\title{
A Novel miRNA Y-56 Mediates the Proliferation of Porcine Skeletal Muscle Satellite Cells by Targeting IGF-1R
}

Jie Song

Jilin University

Linlin Hao

Jilin University

Xiangfang Zeng

China Agricultural University

Rui Yang

Jilin University

Shiyan Qiao

China Agricultural University

Chunli Wang

Jilin University

Hao Yu

Jilin University

Siyao Wang

Jilin University

Yingying Jiao

Jilin University

Hongyao Jia

Jilin University

Songcai Liu ( $\sim$ songcai@jlu.edu.cn )

Jilin University

\section{Research Article}

Keywords: Y-56, IGF-1R, skeletal muscle satellite cells, proliferation

Posted Date: May 27th, 2021

DOI: https://doi.org/10.21203/rs.3.rs-490213/v2 
License: (c) (i) This work is licensed under a Creative Commons Attribution 4.0 International License. Read Full License 


\section{Abstract}

\section{Background:}

The skeletal muscle phenotype of the Bama Xiang pig (BM) is significantly different from the Landrace pig (LP). Uncovering the mechanism of porcine skeletal muscle growth will be of great significance to elucidate the mechanism of the different formation. As the key post-transcriptional regulators, miRNAs play an indispensable role in skeletal muscle development. The proliferation of skeletal muscle satellite cells not only maintain the muscle stem cell population, but also provide a large number of muscle derived cells. Thus, the goal of this study is to explore the effects of a novel miRNA Y-56 on the porcine skeletal muscle satellite cells (PSCs).

\section{Results:}

Firstly, we found that Y-56 was highly expressed in porcine muscle tissues, and its expression was higher in the BM than the LP. The EdU staining and CCK-8 assays results showed that increased levels of Y-56 suppressed cell proliferation, whereas decreased levels of $\mathrm{Y}-56$ resulted in the opposite consequences. Furthermore, flow cytometry results showed that overexpression of $\mathrm{Y}-56$ significantly reduced the percentage of S-phase cells, and the qRT-PCR and western blotting results showed that the expression levels of cyclin dependent kinase 2 (CDK4), proliferating cell nuclear antigen (PCNA) and Cyclin D1 were significantly inhibited. Moreover, downregulation of $\mathrm{Y}-56$ increased the number of S-phase cells and the expression of CDK, PCNA and Cyclin D1. Furtherly, we identified that insulin like growth factor-1 receptor (IGF-1R) was a direct target of Y-56. Consistently, overexpression of IGF-1R promoted the cell proliferation of the PSCs, and increased the number of S-phase cells, as well as up-regulated the expressions of CDK4, PCNA and Cyclin D1. Meanwhile, knock-down of IGF-1R was associated with the opposite tend. Finally, overexpression of IGF-1R partially reversed the inhibition of cell proliferation of the PSCs, the decrease of the percentage of S-phase cells and down-regulation of the expression levels of CDK4, PCNA and Cyclin D1, which caused by overexpression of $\mathrm{Y}-56$.

\section{Conclusion:}

Collectively, our findings suggested that Y-56 represses proliferation and cell cycle process of the PSCs through several biological mechanisms involving downregulation of IGF-1R.

\section{Background}

Being a domesticated mammal, the pig has become an indispensable livestock in people's daily life and excellent medical models because the pig has remarkable similarities with human in body size, anatomical, immunological, physiological, metabolic, dietary habits and evolved similarities [1]. The Bama Xiang pig (BM), a miniature pig from China's Guangxi province, is characterized by short body size, slow growth rate, low lean meat rate and better meat quality, while its skeletal muscle phenotype is significantly different from the Landrace pig (LP) that is characterized by tall body size, fast growth rate, 
high meat yield but poor meat quality [2-3]. Elucidating the relevant mechanism of the different formation of skeletal muscle between the two pig breeds will provide a theoretical basis for improving the porcine production performance and furtherly utilize the pig as medical models. Therefore, understanding the mechanism of porcine skeletal muscle growth will be of great significance to elucidate the mechanism of the formation of the differences between the BM and the LP.

The number of muscle fibers is determined prenatally and remains constant throughout postnatal life, so the growth of skeletal muscle after birth is achieved through the hypertrophy of existing muscle fibers [46]. Studies have shown that the hypertrophy of muscle fibers is related to the increase of DNA content, but the myonuclei do not possess the ability to synthesize DNA [7-8]. Satellite cells are the postnatal source of DNA contributed to growing muscle fibers. DNA accretion occurs through proliferation of satellite cells followed by differentiation and fusion with existing muscle fibers [9]. The proliferation of skeletal muscle satellite cells not only maintain the muscle stem cell population, but also provide a large number of muscle derived cells [10-11]. Therefore, skeletal muscle satellite cells as muscle stem cells play an indispensable role in the growth of skeletal muscle after birth.

MicroRNAs (miRNAs) are conserved, non-coding and endogenous single-stranded RNA molecules involved in the regulation of gene expression in organisms, whose length is generally about 20-24nt. They inhibit translation or promote mRNA degradation generally by associating with the $3^{\prime}$ UTR of their regulatory target mRNAs [12]. Previous studies have identified many miRNAs are the key regulators of skeletal muscle growth [13-14]. According to the expression levels in skeletal muscle and other tissues, miRNAs are divided into two categories. One is the skeletal muscle-specific miRNAs, such as miR-1, miR206 and miR-133, which play an important role in muscle augmentation and differentiation of skeletal muscle satellite cells and myoblasts [15-16]. The other is the non-skeletal muscle-specific miRNA, such as miR-181, miR-34b, miR-26 [17-19]. Research has found that IGF-1 stimulated the up-regulation of miR133, while miR-133 in turn repressed IGF-1R expression to modulate the IGF-1R signaling pathway during skeletal myogenesis [20]. Rui-Song Ye et al. acquired the miRNA and mRNA expression profiles of anterior pituitaries from the BM and the LP by miRNA microarrays and mRNA-seq.Among them, Y-56 is a newly discovered miRNA with high expression in the pig, and no relevant studies have been conducted on it [21].

According to the above analysis, the objective of this study is to assess the expression level of Y-56 in various porcine tissues and to investigate its influences on porcine skeletal muscle growth. We will observe the effects of overexpression of Y-56 on proliferation of porcine skeletal muscle satellite cells and will explore the relevant mechanism, which will lay the foundation for understanding the mechanism of the different formation between the BM and the LP.

\section{Methods}

\section{Animals and cells}

The muscle, heart, liver, spleen, kindey and cartilage tissues were collected from the female Bama Xiang pig (BM) and the Landrace pig (LP) at 7-days age, respectively. The porcine skeletal muscle satellite cells 
(PSCs) were isolated from porcine leg muscle tissues at 7 days of age, following the method [22]. Briefly, the collected leg muscle tissues were washed with PBS supplement with $0.5 \%$ penicillin/streptomycin, then the sterile muscle tissues were minced into small pieces and were digested with collagenase II (Gibco, USA) at $37^{\circ} \mathrm{C}$ for $3-6 \mathrm{~h}$. The suspension was filtered through a $70 \mu \mathrm{m}$ cell strainer and centrifuged at $3000 \mathrm{rpm}$ for $15 \mathrm{~min}$ at room temperature, then we resuspended precipitation by PBS, and filtered by $40 \mu \mathrm{m}$ again, next centrifuged at $3000 \mathrm{rpm}$ for $15 \mathrm{~min}$. After removing the supernatant, we resuspended precipitation by complete culture medium, next the cells was transferred to the culture dish. To separate and purify satellite cells, after $2 \mathrm{~h}$, the suspension was transferred to new culture dish. All animal experiments were performed in accordance with the rules and regulations of the Animal Care and Experimentation Committee of Jilin University (Changchun, China).

\section{Cell Culture}

The 293T cells were cultured in Dulbecco's Modified Eagle Medium (DMEM, Sigma, USA) supplemented with $10 \% \mathrm{FBS}\left(\mathrm{BI}\right.$, Israel) and $0.5 \%$ penicillin/streptomycin (Gibco, USA) in an incubator with $5 \% \mathrm{CO}_{2}$ at $37^{\circ} \mathrm{C}$. The PSCs were cultured in Dulbecco's Modified Eagle Medium/Nutrient Mixture F-12 (DMEM/F-12, Sigma, USA) with $20 \% \mathrm{FBS}, 0.5 \%$ penicillin/streptomycin and $0.5 \%$ chick embryo extract (CEE) and were cultured in an incubator with $5 \% \mathrm{CO}_{2}$ at $37^{\circ} \mathrm{C}$.

\section{Immunofluorescence Staining}

The PSCs cultured in 96-well plates were first treated with $4 \%$ formaldehyde for 15 min and then permeabilized by $0.1 \%$ Triton X-100. Next, after being blocked in $10 \%$ FBS for 30 min, the cells were sequentially incubated overnight with anti-Pax7 (Abcom, US; 1:100). Thereafter, the cells were treated with an Alexa Fluor 594-conjugated anti-mouse IgG (Bioworld Technology, USA; 1:200) according to the manufacturer's instructions. Finally, the nuclei were stained with DAPI (Beyotime, Shanghai, China) for 5 $\mathrm{min}$. Then the images were captured with an inverted luorescence microscope (Nikon, Japan) and the data were analyzed in Image $\mathrm{J}$ software (National Institutes of Health, Bethesda, MD, USA).

\section{Plasmid Construction and Cell Transfection}

The psiCHECK-2 dual-luciferase reporter vector: The 3'UTR fragment of IGF-1R containing the Y-56 binding site was cloned into the psiCHECK-2 dual-luciferase reporter vector and the fragment named IGF1R-WT and IGF-1R-MT were synthesized by Genewiz (Beijing, China). The sequences are listed in Table 1.

The IGF-1R overexpression vector: The full coding sequence (CDS) of IGF-1R was synthesized by Genewiz (Beijing, China) and cloned into the pcDNA3.1(+) expression vector.

The RNA oligonucleotides used in this study, including Y-56 mimics, mimics-NC, Y-56 inhibitor, inhibitorNC, si-IGF-1R and si-NC, were obtained from Gene Pharma (Shanghai, China) and were shown in Table 1. The oligonucleotides and plasmids were transfected using Lipofectamine 2000 reagent (Invitrogen, USA) according to the manufacturer's protocol with at least three replications. 
Table 1 Oligonucleotide sequences used in this study

\begin{tabular}{|ll|}
\hline Fragment Name & Sequence (5' to 3' $\left.^{\prime}\right)$ \\
\hline Y-56 mimics & AGAGGAGGAAGAGGAGGAGGA \\
mimics-NC & CUCCUCCUCUUCCUCCUCUUU \\
& ACGUGACACGUUCGGAGAATT \\
\hline Y-56 inhibitor & UCCUCCUCCUCUUCCUCCUCU \\
\hline inhibitor-NC & CAGUACUUUUGUGUAGUACAA \\
\hline Si-IGF-1R & CCUCGAGCUAGAGAACUUTT \\
& AAGUUCUCUAGCUCCGAGGTT \\
\hline
\end{tabular}

\section{Dual-Luciferase Reporter Assay}

The psiCHECK-2 dual-luciferase reporter vector (200 ng) containing a wild-type or mutant IGF-1R 3'UTR fragment was co-transfected with Y-56 mimics or mimics-NC duplexes (100 nM) into 293T cells in a 96well plate with six independent repeats. After $48 \mathrm{~h}$ of transfection, the Firefly and Renilla luciferase activities were measured in a Fluorescence/Multi-Detection Microplate Reader (BioTek, Winooski, VT, USA) using a Dual-GLO Luciferase Assay System Kit (Promega, Madison, WI, USA).

\section{Quantitative real-time PCR}

Total RNA was isolated from the collected tissues and the PSCs after transfection using RNAiso Plus (Takara, Ostu, Japan). cDNA synthesis for mRNA was carried out using the PrimeScript RT reagent Kit (Takara, Japan). The reverse transcription reaction for miRNA was performed with the PrimeScript RT reagent Kit (Takara, Japan) using specific Bulge-loop miRNA qRT-PCR Primer for Y-56 and U6 that were synthesized by Genewiz (Beijing, China). qRT-PCR reactions were carried out with the SYBR Green PCR Master Mix (Thermo Fisher Scientific, MA) according to the manufacturer's instructions on an ABI PRISM $7900 \mathrm{HT}$ thermocycler (Applied Biosystems, Foster City, CA, USA). The comparative $\mathrm{Ct}\left(2^{-\Delta \mathrm{Ct}}\right)$ method was used for qRT-PCR data analysis. All amplifications were performed in triplicate, and the mRNA level of the target genes was normalized to the GAPDH gene as an internal reference. The primers used for qRT-PCR are listed in Table 2.

Table 2. Primers used in this study 


\begin{tabular}{|lll|}
\hline Primer Name & Primer Sequences (5'to ${ }^{\prime}$ ') & Size (bp) \\
\hline IGF-1R & F: TGCCCTTCAGGCTTCATC & 91 \\
& R: TCTTCTCTTCCTCACAGACTTTG & \\
CDK4 & F: TTCGAGCATCCCAATGTTGTC & 225 \\
& R: GTCTCGATGAACGATGCAGTTG & \\
PCNA & CAATTTGGCCATGGGCGTGA & 103 \\
Cyclin D1 & FGTGTCTGCATTATCTTCTGCC & \\
GAPDH TGCATCTACACCGACAACTCCA & 222 \\
& R: GTTGGAAATGAACTTCACGTCTGT & \\
\hline U6 TCGGAGTGAACGGATTTGGC & 189 \\
& R: TGACAAGCTTCCCGTTCTCC & \\
\hline
\end{tabular}

\section{Cell counting kit-8 assay}

The cell proliferation rate of the PSCs was assessed at $0 \mathrm{~h}, 24 \mathrm{~h}, 48 \mathrm{~h}, 72 \mathrm{~h}$ and $96 \mathrm{~h}$ after transfection using a Cell Counting Kit-8 (CCK-8) (Beyotime, China). Briefly, the PSCs were seeded in a 96-well format at a density of 5000 cells/well and were then transfected with different miRNAs, vectors or siRNAs as described above. $10 \mu \mathrm{L}$ of CCK-8 solution was then added to each well containing $100 \mu \mathrm{L}$ of the culture medium and then the plate was incubated for $30 \mathrm{~min}$ at $37^{\circ} \mathrm{C}$, and the absorbance was read at a wavelength of $450 \mathrm{~nm}$ using a microplate reader (TECAN, Switzerland).

\section{EdU staining}

The PSCs were seeded in 96-well plates at a concentration of $2 \times 10^{3}$ per well. Then the PSCs were treated with Y-56 mimics, Y-56inhibitor, pcDNA3.1+IGF1R and si-IGF-1R for $48 \mathrm{~h}$ and incubated with 50 $\mu \mathrm{M}$ EDU (RiboBio, Guangzhou, China) for $3 \mathrm{~h}$. The cells were washed twice with PBS, then were fixed with $4 \%$ paraformaldehyde for $15 \mathrm{~min}$ and were permeabilized with $0.5 \%$ Trixon- 100 for $20 \mathrm{~min}$. At the end of each step, the cells were washed twice with PBS for $5 \mathrm{~min}$. According to the kit, the cells were incubated in a mixture of Reagents for $30 \mathrm{~min}$. The nuclei were stained with DAPI for $5 \mathrm{~min}$. The stained cells were finally observed on an inverted luorescence microscope (Nikon, Japan) and the data were analyzed in Image J (National Institutes of Health, Bethesda, MD, USA).

\section{Flow cytometry}


The PSCs were cultured in a 6-well culture plate at a density of $4 \times 10^{5}$ per well and were treated with Y-56 mimics, Y-56inhibitor, pcDNA3.1+IGF1R and si-IGF-1R for $48 \mathrm{~h}$. Then, the cells were digested with $0.25 \%$ trypsin and terminated with DMEMF-12 containing $20 \%$ FBS, next the cells were collected and fixed in cold $70 \%$ ethanol overnight at $4{ }^{\circ} \mathrm{C}$. The cells were then washed twice and stained with $50 \mathrm{mg} / \mathrm{mL}$ propidium iodide $(\mathrm{PI})$ for $30 \mathrm{~min}$. Finally, the cell cycles were analyzed by flow cytometry (Becton Dickinson, USA).

\section{Western blotting analysis}

The PSM cells $48 \mathrm{~h}$ after transfection were harvested to extract the proteins using the lysis buffer (KeyGen Biotech, China) complement with protease and protein phosphatase inhibitor on ice. Protein concentration was determined using BCA Protein Assay Kit (Beyotime, China) and $30 \mu \mathrm{g}$ protein per sample was loaded and separated using a $5 \%$ stacking gel and a $12 \%$ separating gel. Separated proteins were transferred to PVDF membrane, which was then blocked in $5 \% \mathrm{BSA}$ for $2 \mathrm{~h}$ at room temperature and incubated with primary antibodies at $4{ }^{\circ} \mathrm{C}$ overnight. After washing thrice (10 min once) in TBST, membranes were incubated with HRP conjugated goat anti-rabbit IgG or HRP conjugated goat anti-mouse IgG (Bioworld Technology, USA, 1:2000) $2 \mathrm{~h}$ at room temperature.

The Western blot was visualized by Enhanced Chemiluminescence (ECL, Bioworld, USA) performed on Bio-Rad Gel Doc XR instrument (Bio-Rad, Hercules, CA, United States). Thereafter, the gray value of the target strip was analyzed using Geno Sens Analysis software and the expression of the target proteins was analyzed by the ratio of the gray value of the target strip to the gray value of $\beta$-actin.

The primary antibodies used in this study were as follows: IGF-1R rabbit polyclonal antibody (Abcom, US, 1:1000), PCNA (proliferating cell nuclear antigen) rabbit polyclonal antibody, CDK4 (cyclin-dependent kinase 4) rabbit polyclonal antibody, Cyclin D1 rabbit polyclonal antibody (Cell Signaling Technology, USA, 1:2000) and $\beta$-actin polyclonal antibody (BBI, China, 1:2000).

\section{Statistical Analysis}

All experimental results are presented as the mean \pm SEM, with at least three independent replications. The statistically significant difference between groups was tested by one-way ANOVA using GraphPad Prism version 6.01. We considered $P<0.05$ to be statistically significant. * $P<0.05$; $* \star P<0.01$; $* \star \star P<$ 0.001 .

\section{Results}

\section{Y-56 is highly expressed in muscle and is upregulated in the BM compared with LP}

Rui-Song Ye et al. compared the miRNA expression profiles in anterior pituitary between the BM and the LP [21], and the Table S3 data - "All miRNAs expression" were used in this study. For an overview of the differentially expressed miRNAs, a volcano plot was drawn where the fold-change of miRNA expression 
was plotted on the $x$-axis, versus the significance of difference in miRNA expression between pools on the $y$-axis (Fig.1A). Differential expression analysis revealed that 41 miRNAs (32 up and 9 down) were regulated significantly in the $B M$ after FDR correction ( $\nabla$ fold change $\triangle \geq 1.5, P<0.001$ ). Among the 32 upregulated miRNAs, Y-56 was a new miRNA. Then we examined the expression of Y-56 in porcine other tissues and found that $\mathrm{Y}-56$ highly expressed in muscle tissues (Fig.1B). Furthermore, we compared the expression level of $Y-56$ in leg muscle tissues between the LP and the BM, then the results showed that the $\mathrm{Y}-56$ expression in the BM was significantly higher than that in the LP $($ Fig. $1 C)(P<0.001)$.

A. The volcano plot of observed fold changes in miRNA expression vs significance. B. qRT-PCR analysis of $Y-56$ expression in porcine different tissues; $C$. The mRNA expression level of $Y-56$ in the LP and the $\mathrm{BM}$.

\section{Y-56 suppresses proliferation of the PSCs}

Firstly, we isolated the PSCs according to the above description and the PSCs were identified by pax7 (Fig.S1). To reveal the effect of Y-56 on proliferation of the PSCs, we transfected the Y-56 mimics into the PSCs (Fig.2A). The EdU staining assay showed that the number of EdU labeled positive cells reduced in the $\mathrm{Y}-56$ mimics group, unlike in the mimics-NC group (Fig. 2B-C) $(\mathrm{P}<0.01)$. The CCK-8 assay also found that the Y-56 overexpression hampered the proliferation of the PSCs (Fig. 2D). In addition, flow cytometer assays showed that the PSCs numbers were lower in G1-phase $(P<0.01)$, whereas the proportion of the PSCs was increased in the S-phase (Fig.2E-F) $(P<0.01)$. The proteins encoded by CDK4, PCNA and Cyclin D1 have been identified to perform critical functions in $G 1, G 2$ and $S$ phases during cell cycle progression [23-25]. When Y-56 was overexpressed, the results of qRT-PCR and western blotting showed that the expressions of CDK4, PCNA and Cyclin D1 were significantly decreased (Fig. 2G-I).

A. The transfection efficiency of Y-56 mimics; B. EdU staining after the transfection of Y-56 mimic in the PSCs; C. The statistical results of EdU staining; D. The CCK-8 assay after transfecting Y-56 mimics into the PSCs; E. Flow cytometry raw data of cell cycle analysis in the PSCs after overexpression of Y-56; F. The statistical results of cell cycle analysis; $G$. The mRNA expression levels of the cell cycle marker genes;

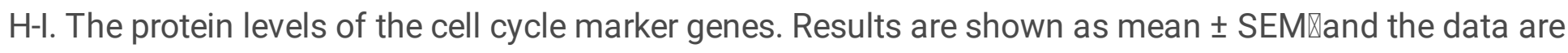
representative of at least three independent assays. Independent sample t-test was used to analyze the statistical differences between groups. ( $\mathrm{P}<0.05$; ${ }^{\star *} \mathrm{P}<0.01$, *** $\left.\mathrm{P}<0.001\right)$.

To further explore the effect of Y-56 on the PSCs proliferation, we next treated the cells with Y-56 inhibitor and inhibitor-NC, and the expression of Y-56 in the Y-56 inhibitor group was dramatically reduced below the inhibitor-NC group (Fig.3A) $(P<0.001)$. The number of EdU positive cells markedly increased in the $Y$ 56 inhibitor group (Fig. 3B-C) $(\mathrm{P}<0.001)$. Our CCK-8 assay also verified the knock-down of Y-56 induced cell viability (Fig. 3D). The flow cytometry assay results indicated down-regulation of the G1-phase and G2-phase cells and up-regulation the S-phase cells after suppressing the expression of Y-56 (Fig. 3E-F). qRT-PCR and western blotting data showed that inhibition of Y-56 depressed the expression of CDK4, PCNA and Cyclin D1 (Fig. 3G-I). Therefore, we suggested that the overexpression of Y-56 suppressed the PSCs proliferation, while knock-down of Y-56 promoted the PSCs proliferation. 
A. The transfection efficiency of Y-56 inhibitor; B. EdU staining after the transfection of Y-56 inhibitor in the PSCs; C. The statistical results of EdU staining; D. The CCK-8 assay after transfecting Y-56 inhibitor into the PSCs; E. Flow cytometry raw data of cell cycle analysis in the PSCs after inhibition of Y-56; F. The statistical results of cell cycle analysis; G. The mRNA expression levels of the cell cycle marker genes; $\mathrm{H}-\mathrm{I}$. The protein levels of the cell cycle marker genes. Results are shown as mean \pm SEMヌand the data are representative of at least three independent assays. Independent sample t-test was used to analyze the statistical differences between groups. ( $P<0.05 ; * \star P<0.01$, $* \star \star P<0.001)$.

\section{IGF-1R is a direct target gene of Y-56}

The experiments described above showed that Y-56 can promote the PSCs proliferation (Fig.2 and Fig.3). To better understand the regulatory mechanism of this process, we used TargetScan7.2 and miRTar Base to predict potential target genes. We detected IGF-1R as a candidate target gene and the seed sequence of $\mathrm{Y}-56$ was presented in Fig. 4A. The secondary structures formed by $\mathrm{Y}-56$ binding to the 3'UTR of IGF-1R was shown in Fig. 4B, as well as the corresponding minimum free energy formed by $\mathrm{Y}-56$ and IGF-1R was $-27.4 \mathrm{kcal} / \mathrm{mol}$. Then we constructed wild-type IGF-1R 3'UTR (IGF-1R-WT) and mutant IGF-1R 3'UTR (IGF1R-MT) dual luciferase reporter vectors accordingly. Then the mRNA expression levels of IGF-1R in porcine different tissues were detected, and the results showed that IGF-1R mRNA expression level was negatively correlated with Y-56 (Fig. 4C and Fig. 1B). Next, we found that the dual-luciferase activity of IGF-1R-WT and Y-56 mimics co-transfected into the 293T cells was higher than that of co-transfected IGF1R-WT and mimics-NC, while the dual-luciferase activity of IGF-1R-MT with Y-56 mimics and mimics-NC appeared to have no effect (Fig. 4D) $(P<0.01)$. To further verify that $Y-56$ targets the 3'UTR of endogenous IGF-1R, Y-56 mimics were transfected into the PSCs. The results of qRT-PCR and western blotting data suggested that IGF-1R mRNA and protein levels were reduced and increased, respectively, in $\mathrm{Y}-56$ mimics group and mimics-NC group (Fig. 4E-G) $(\mathrm{P}<0.01)$.

A. The Y-56 binding site in the IGF-1R mRNA 3'UTR; B. The potential secondary structures formed by Y-56 binding to IGF-1R mRNA 3'UTR; C. The relative mRNA expression of IGF-1R in porcine different tissues; D. The dual-luciferase reporter assay was performed by co-transfecting a wild-type or mutant IGF-1R 3'UTR with Y-56 mimic or mimic NC in 293T cells; E-G. The mRNA and protein levels of IGF-1R in the PSCs after being transfected with Y-56 mimic or Y-56 inhibitor. Results are shown as mean \pm SEM खand the data are representative of at least three independent assays. Independent sample t-test was used to analyze the statistical differences between groups. ( $\mathrm{P}<0.05$; $* * \mathrm{P}<0.01)$.

\section{IGF-1R promotes the cell proliferation of the PSCs}

To address the function of IGF-1R in the regulation of the PSCs proliferation, the pcDNA3.1+IGF-1R expression vector was constructed, and the mRNA expression in the pcDNA3.1+IGF-1R group was higher than the pcDNA3.1 group (Fig. 5A) $(P<0.001)$. The EdU staining and CCK-8 assays were carried out to evaluate the PSCs proliferation, and the results showed that the number of cells in the proliferative phase was significantly increased due to the overexpression of IGF-1R (Fig. 5B-C and Fig. 5D) $(P<0.001)$. The number of cells in G1-phase and G2-phase were less than that in the control group after IGF-1R 
overexpression $(P<0.01)$, while the S-phase cells number were higher (Fig. 5E-F) $(P<0.01)$. Furthermore, the analysis of q-RT-PCR and western blotting found that overexpression of IGF-1R also significantly increased CDK4, PCNA and Cyclin D1 expression at both mRNA and protein levels (Fig. 5G-I) $(P<0.01)$.
A. The mRNA level of IGF-1R after IGF-1R overexpression; B-C. The proliferation rate of the PSCs after overexpression of IGF-1R by EdU assay; D. The CCK-8 assay after overexpression of IGF-1R in the PSCs; E- F. Flow cytometry raw data and statistical results of cell cycle analysis after IGF-1R overexpression; G-H. The mRNA and protein expression of the cell cycle marker genes. Results are shown as mean \pm SEM $\mathbb{\text { and }}$ the data are representative of at least three independent assays. Independent sample t-test was used to analyze the statistical differences between groups. ( $P<0.05$; $* * 20.01$; $* \star * ~ P<0.001$ ).

Next, we employed siRNA technology to confirm the role of IGF-1R in the PSCs. It showed that si-IGF-1R down-regulated the expression of IGF-1R efficiently at the mRNA (Fig. 6A) $(P<0.001)$. The EdU assay found that the inhibition of IGF-1R could hamper the proliferation of the PSCs (Fig. 6B-C) and the CCK-8 assay showed the same results (Fig. 6D). In addition, the cells in G1-phase were significantly increased after being transfected with si-IGF-1R, but the number cells of the S-phase and G2-phase were noteworthy decreased (Fig. 6E-F) $(P<0.01)$. What's more, when the si-IGF-1R was transfected into the PSCs, the expression of CDK4, PCNA and Cyclin D1 were suppressed at both mRNA and protein levels (Fig. 5G-I) (P $<0.01$ ). Collectively, these results showed a opposite trend with overexpression of IGF-1R, and all proofs demonstrated that IGF-1R could promote the cell proliferation of the PSCs.

A. The mRNA level of IGF-1R after the transfection of si-IGF-1R in the PSCs; B-C. EdU staining assay of the PSCs after IGF-1R knockdown; D. The CCK-8 assay after inhibition of IGF-1R in the PSCs; E-F. Flow cytometry raw data and statistical results of cell cycle analysis after IGF-1R knockdown; G-H. The mRNA and protein expression of the cell cycle marker genes. Results are shown as mean \pm SEMXand the data are representative of at least three independent assays. Independent sample t-test was used to analyze the statistical differences between groups. ( $\left.{ }^{\star} P<0.05 ; * \star P<0.01\right)$.

\section{Overexpression of IGF-1R partially reverses the inhibitory effect of Y-56 on the PSCs}

To verify that Y-56 regulated cell proliferation of the PSCs by IGF-1R furtherly, we co-transfected pcDNA3.1+IGF-1R and Y-56 mimics. The EdU and CCK-8 assays both proved the decreased proliferation capabilities of the PSCs caused by Y-56 overexpression were retarded by overexpression of IGF-1R (Fig. $7 A-C)(P<0.01)$. Meanwhile, the cell cycle changes also showed that IGF-1R overexpression reversed the inhibition by $Y-56$ mimics (Fig. 7D-E) $(P<0.01)$, and the mRNA and protein expression levels of CDK4, PCNA and Cyclin D1showed the same trend (Fig. 7F-H) $(P<0.001)$. Collectively, our data suggest that the suppressing role of Y-56 in maintaining the proliferation of the PSCs was largely dependent on the IGF$1 \mathrm{R}$.

A-B. EdU staining assay of the PSCs after co-transfection of IGF-1R and Y-56 mimics; C. The CCK-8 assay after o-transfection of IGF-1R and Y-56 mimics in the PSCs; D-E. Flow cytometry raw data and statistical results of cell cycle analysis after co-transfection of IGF-1R and Y-56 mimics; G-H. The mRNA and protein 
expression of the cell cycle marker genes after co-transfection of IGF-1R and Y-56 mimics in the PSCs.

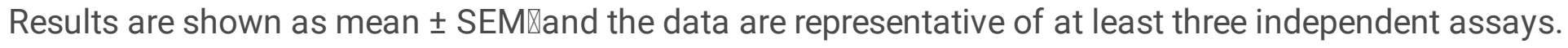
Independent sample t-test was used to analyze the statistical differences between groups. $\left({ }^{*} P<0.05 ; * \star\right.$ $P<0.01 ; * \star \star P<0.001)$.

\section{Discussion}

The BM (Bama Xiang pig) has obvious growth difference with the LP (Landrace pig). As a local breed in China, the BM has short size, slow growth rate but better meat quality, and it is an important model for medical experiment [1-3]. Uncovering the relevant mechanism of the differences between the two breeds will provide favorable basic evidence for the future porcine industry and even the medical industry. Skeletal muscle, an important tissue that accounts for $40-60 \%$ of the animal body, plays an indispensable role in the growth and development of animals after birth [26]. miRNAs have been proved to be the key factor to regulate the growth and development of skeletal muscle [27]. In the prenatal skeletal muscles of Landrace, Tongcheng and Wuzhishan pigs, miRNA-133b and miRNA-206 had different expression patterns, which indicated that the same miRNAs would play regulatory mechanisms in different genotype pigs [28]. Skeletal muscle satellite cells, as muscle stem cells, are an important cell source of muscle development after birth [8-9]. Skeletal muscle satellite cells bear on the formation of muscle in postpartum animals, they go through the cell proliferation, differentiation and fusion to form muscle, finally $[11,29]$. Therefore, its proliferation ability will directly affect its differentiation and fusion. This is the reason why this paper focused on exploring the effect of a novel miRNA on the proliferation of skeletal muscle satellite cells during the development of porcine.

Many miRNAs were proved to be involved in the regulation of skeletal myogenesis by binding to its target genes, for examples, miR-34c inhibits cell proliferation of the PSCs but promotes the differentiation by targeting Notch 1 [30], and miR-199b has an inhibitory effect on the proliferation of muscle satellite cells in Large White pigs by targeting the JAG1 gene [31], as well as miR-27b promotes muscle development by inhibiting MDFI expression [32]. Similar as previously reported, Our EdU staining and CCK-8 assays showed that the novel miRNA Y-56 had the ability to inhibit the proliferation of skeletal muscle satellite cells by targeting IGF-1R gene (Fig. 2-4). However, the effect of Y-56 on the differentiation ability of skeletal muscle satellite cells remains to be further explored.

In addition, the cell proliferation of PSCs is dependent on an active cell cycle [31]. The results of flow cytometry showed that high expression levels of Y-56 also increased the percentage of cells in the G1phase and reduced the number of cells in S-phase by inhibiting the expression of IGF-1R (Fig. 2E and Fig. $5 \mathrm{E}$ ). In the cell cycle, CDK4 is a kinase that regulates the transition from the $\mathrm{G} 1$ to $S$ phases of the cell cycle [33], and PCNA is a key factor in DNA replication and cell cycle regulation [34], as well as Cyclin D1 is proposed to serve as an active switch in the regulation of continued cell cycle progression [35]. Consistently, the mRNA and protein expression levels of CDK4, PCNA and Cyclin D1 were all shown to be downregulated when overexpressing Y-56, while these marker genes were upregulated when the 
expression of IGF-1R was increased in the PSCs. Thus, Y-56 inhibit the expression levels of cell cycle factors: CDK4, PCNA and Cyclin D1 by targeting IGF-1R gene to suppress the cycle process of the PSCs.

IGF-1R is an important receptor of IGF-1, while IGF-1 has been proved to have a positive effect on the growth and development of the skeletal muscle [36-37]. Meanwhile, IGF-1R is required for cell growth and development and to maintain the cell cycle [38-40]. Substantial pieces of direct evidence have emerged that the biological function of IGF-1R is generally achieved by activating two of the following most important intracellular signaling pathways: MAPK/ERK and PI3K/AKT after binding to the ligand IGF-1 [41-42]. In our previous study, we found that IGF-1R promotes the cell proliferation on the PK-15 cells and IGF-1R promotes $\mathrm{C} 2 \mathrm{C} 12$ proliferation through the AKT and ERK signaling pathways [43-44]. There have been no such results regarding the function of IGF-1R on the PSCs published previously, however, our results demonstrate the proliferation-promoting function of IGF-1R on the PSCs, as well as, overexpression of IGF-1R increases the cell cycle and up-regulates the marker genes (CDK4, PCNA and Cyclin D1) expression levels (Fig. 5-6).

\section{Conclusions}

Y-56 reduces the mRNA and protein expression levels of IGF-1R by targeting IGF-1R mRNA 3'UTR, meanwhile, overexpression of Y-56 inhibits the expression of cell cycle-related genes (CDK4, PCNA and Cyclin D1), leading to the blocked cell cycle process and the impeding of the PSCs proliferation, finally (Fig. 8). In summary, our study provides new insight into understanding the mechanisms of the different formation of the skeletal muscle growth between the BM and the LP.

\section{Abbreviations}

IGF-1R: insulin like growth factors-1 receptor; 3'UTR: 3'-untranslated region; PSCs: porcine skeletal muscle satellite cells; qRT-PCR: quantificational real-time polymerase chain reaction; CDK4: Cyclin dependent kinase 4; PCNA: Proliferating cell nuclear antigen; Cyclin D1: Cell cycle protein D1.

\section{Declarations}

\section{Ethics approval and consent to participate}

Animal experimental procedures were approved by the Institutional Animal Care and Use Committee of Jilin University (Changchun, China, Approval number SY201912002, 2 December 2019). All animal welfare and experimental procedures were performed strictly according to the guidelines from the National Institutes of Health Guide for the Care and Use of Laboratory Animals (NIH Publications No. 8023, revised 1978).

\section{Consent for publication}

Not applicable. 
Availability of data and material

The data sets used and/or analyzed during the current study are available from the corresponding author on reasonable request

\section{Competing interests}

The authors declare that they have no competing interests.

\section{Funding}

This work was supported by the National Natural Science Foundation of China (31772699).

\section{Authors' contributions}

JS, LLH, SCL and HYJ conceived and designed the experiments. JS, RY performed the experiments. JS, XFZ, CLW and HY analyzed the data. SYQ, SYW and YYJ contributed to the sample collections and helped to facilitate the experimental process. JS wrote the manuscript, then LLH, SCL and HYJ modified the manuscript. The authors read and approved the final manuscript.

\section{Acknowledgements}

We are grateful to our laboratory members for helpful comments.

\section{Authors' information}

1 College of Animal Science, Jilin University, 5333 Xi'an Road, Changchun, Jilin 130062, China

2 State Key Laboratory of Animal Nutrition, College of Animal Science and Technology, China Agricultural University, Beijing 100193, China

3 Ministry of Health Key Laboratory of Radiobiology, College of Public Health, Jilin University, Changchun, Jilin 130021, China

\section{References}

[1] Dominique Rocha,Graham Plastow. Commercial pigs: an untapped resource for human obesity research? [J]. Drug Discovery Today,2006,11(11).

[2] Kim Gap-Don,Yang Han-Sul,Jeong Jin-Yeon. Intramuscular variations of proteome and muscle fiber type distribution in semimembranosus and semitendinosus muscles associated with pork quality[J]. Food chemistry,2018,244.

[3] Kahane N,Cinnamon Y,Kalcheim C. The cellular mechanism by which the dermomyotome contributes to the second wave of myotome development[J]. Development (Cambridge, England),1998,125(21). 
[4] Stickland N C,Widdowson E M,Goldspink G. Effects of severe energy and protein deficiencies on the fibres and nuclei in skeletal muscle of pigs[J]. The British journal of nutrition,1975,34(3).

[5] Wigmore P M,Strickland N C. DNA, RNA and protein in skeletal muscle of large and small pig fetuses[J]. Growth,1983,47(1).

[6] Wigmore P M, Stickland N C. Muscle development in large and small pig fetuses[J]. Journal of anatomy,1983,137 (Pt 2).

[7] Stockdale F.E., Holtzer H. DNA synthesis and myogenesis[J]. Academic Press,1961,24(3).

[8] Mesires NT, Doumit ME. Satellite cell proliferation and differentiation during postnatal growth of porcine skeletal muscle[J]. American Journal of Physiology-Cell Physiology. 2002, 282(4):C899-906.

[9] Moss F P,Leblond C P. Satellite cells as the source of nuclei in muscles of growing rats. [J]. The Anatomical record,1971,170(4).

[10] Campion D R,Richardson R L,Reagan J O,Kraeling R R. Changes in the satellite cell population during postnatal growth of pig skeletal muscle[J]. Journal of animal science,1981,52(5).

[11] Yin H, Price F, Rudnicki MA. Satellite cells and the muscle stem cell niche[J]. Physiological Reviews. 2013, 93(1): 23-67.

[12] Liu Jidong,Valencia-Sanchez Marco Antonio,Hannon Gregory J,Parker Roy. MicroRNA-dependent localization of targeted mRNAs to mammalian P-bodies[J]. Nature cell biology,2005,7(7).

[13] David P. Bartel. MicroRNAs: Target Recognition and Regulatory Functions[J]. Cell,2009,136(2).

[14] Buckingham M, Relaix F. The role of Pax genes in the development of tissues and organs: Pax3 and Pax7 regulate muscle progenitor cell functions[J]. Annual Review of Cell and Developmental Biology, 2007, 23:645-73.

[15] Wüst Stas,Dröse Stefan,Heidler Juliana,Wittig Ilka,Klockner Ina,Franko Andras,Bonke Erik,Günther Stefan,Gärtner Ulrich,Boettger Thomas,Braun Thomas. Metabolic Maturation during Muscle Stem Cell Differentiation Is Achieved by miR-1/133a-Mediated Inhibition of the Dlk1-Dio3 Mega Gene Cluster[J]. Cell metabolism,2018,27(5).

[16] Ma Guoda,Wang Yajun,Li You,Cui Lili,Zhao Yujuan,Zhao Bin,Li Keshen. MiR-206, a key modulator of skeletal muscle development and disease[J]. International journal of biological sciences,2015,11(3).

[17] Naguibneva I, Ameyar-Zazoua M, Polesskaya A, Ait-Si-Ali S, Groisman R, Souidi M, Cuvellier S, HarelBellan A. The microRNA miR-181 targets the homeobox protein Hox-A11 during mammalian myoblast differentiation[J]. Nature Cell biology. 2006, 8(3):278-84. 
[18] Wang Zhijun,Zhang Xiaocui,Li Zhenhui,Abdalla Bahareldin Ali,Chen Yangfeng,Nie Qinghua. MiR-34b$5 p$ Mediates the Proliferation and Differentiation of Myoblasts by Targeting IGFBP2. [J]. Cells,2019,8(4).

[19] Luo, Xiaobin,Pan, Zhenwei,Shan, Hongli,Xiao, Jiening,Sun, Xuelin,Wang, Ning,Lin, Huixian,Xiao, Ling,Maguy, Ange,Qi, Xiao-Yan,Li, Yue,Gao, Xu,Dong, Deli,Zhang, Yong,Bai, Yunlong,Ai, Jing,Sun, Lihua,Lu, Hang,Luo, Xiao-Yan,Wang, Zhiguo,Lu, Yanjie,Yang, Baofeng,Nattel, Stanley. MicroRNA-26 governs profibrillatory inward-rectifier potassium current changes in atrial fibrillation[J]. Journal of Clinical Investigation,2013,123(5).

[20] Huang Mian-Bo,Xu Hui,Xie Shu-Juan,Zhou Hui,Qu Liang-Hu. Insulin-like growth factor-1 receptor is regulated by microRNA-133 during skeletal myogenesis[J]. PloS one,2011,6(12).

[21] Rui-Song Ye,Meng Li,Qi-En Qi,Xiao Cheng,Ting Chen,Chao-Yun Li,Song-Bo Wang,Gang Shu,Li-Na Wang,Xiao-Tong Zhu,Qing-Yan Jiang,Qian-Yun Xi,Yong-Liang Zhang. Comparative Anterior Pituitary miRNA and mRNA Expression Profiles of Bama Minipigs and Landrace Pigs Reveal Potential Molecular Network Involved in Animal Postnatal Growth[J]. PLOS ONE,2015,10(7).

[22] Li Long,Cheng Xiaofang,Chen Ling,Li Jingxuan,Luo Wenzhe,Li Changchun. Long Noncoding Ribonucleic Acid MSTRG.59589 Promotes Porcine Skeletal Muscle Satellite Cells Differentiation by Enhancing the Function of PALLD[J]. Frontiers in genetics,2019,10.

[23] Lindsey R Pack,Leighton H Daigh,Tobias Meyer. Putting the brakes on the cell cycle: mechanisms of cellular growth arrest[J]. Current Opinion in Cell Biology,2019,60.

[24] S. N. Naryzhny. Proliferating cell nuclear antigen: a proteomics view[J]. Cellular and Molecular Life Sciences,2009,65(23).

[25] James A Coffman. Cell Cycle Development[J]. Developmental Cell,2004,6(3).

[26] Caroline W. Beck,Bea Christen,Donna Barker,Jonathan M.W. Slack. Temporal requirement for bone morphogenetic proteins in regeneration of the tail and limb of Xenopus tadpoles[J]. Mechanisms of Development,2006,123(9).

[27] David P. Bartel. MicroRNAs: Target Recognition and Regulatory Functions[J]. Cell,2009,136(2).

[28] Tang Zhonglin,Yang Yalan,Wang Zishuai,Zhao Shuanping,Mu Yulian,Li Kui. Integrated analysis of miRNA and mRNA paired expression profiling of prenatal skeletal muscle development in three genotype pigs[J]. Scientific reports,2015,5.

[29] Alexander Mauro. Satellite Cell of Skeletal Muscle Fibers[J]. The Journal of Biophysical and Biochemical Cytology,1961,9(2).

[30] Lianjie Hou,Jian Xu,Huaqin Li,Jinxin Ou,Yiren Jiao,Chingyuan Hu,Chong Wang. MiR-34c represses muscle development by forming a regulatory loop with Notch1[J]. Scientific Reports,2017,7(1). 
[31] Linhui Zhu,Lianjie Hou,Jinxin Ou,Guli Xu,Fangyi Jiang,Chingyuan Hu,Chong Wang. MiR-199b represses porcine muscle satellite cells proliferation by targeting JAG1[J]. Gene,2018,691.

[32] Lianjie Hou,Jian Xu,Yiren Jiao,Huaqin Li,Zhicheng Pan,Junli Duan,Ting Gu,Chingyuan Hu,Chong Wang. MiR-27b Promotes Muscle Development by Inhibiting MDFI Expression[J]. Cellular Physiology and Biochemistry,2018,46(6).

[33] Alice Grison,Carine Gaiser,Andrea Bieder,Constanze Baranek,Suzana Atanasoski. Ablation of cdk4 and cdk6 affects proliferation of basal progenitor cells in the developing dorsal and ventral forebrain[J]. Developmental Neurobiology,2018,78(7).

[34] Wojciech Strzalka,Alicja Ziemienowicz. Proliferating cell nuclear antigen (PCNA): a key factor in DNA replication and cell cycle regulation[J]. Annals of Botany,2011,107(7).

[35] Dennis W Stacey. Cyclin D1 serves as a cell cycle regulatory switch in actively proliferating cells[J]. Current Opinion in Cell Biology,2003,15(2).

[36] Yoshida Tadashi,Delafontaine Patrice. Mechanisms of IGF-1-Mediated Regulation of Skeletal Muscle Hypertrophy and Atrophy[J]. Cells,2020,9(9).

[37] Ahmad Syed Sayeed,Ahmad Khurshid,Lee Eun Ju,Lee YongHo,Choi Inho. Implications of Insulin-Like Growth Factor-1 in Skeletal Muscle and Various Diseases[J]. Cells,2020,9(8).

[38] Carrasco-Garcia Estefania,Martinez-Lacaci Isabel,Mayor-López Leticia,Tristante Elena,CarballoSantana Mar,García-Morales Pilar,Ventero Martin Maria Paz,Fuentes-Baile Maria,Rodriguez-Lescure Álvaro,Saceda Miguel. PDGFR and IGF-1R Inhibitors Induce a G2/M Arrest and Subsequent Cell Death in Human Glioblastoma Cell Lines[J]. Cells,2018,7(9).

[39] Yingbo Lin,Hongyu Liu,Ahmed Waraky,Felix Haglund,Prasoon Agarwal,Helena Jernberg-Wiklund,Dudi Warsito,Olle Larsson. SUMO-modified insulin-like growth factor 1 receptor (IGF-1R) increases cell cycle progression and cell proliferation[J]. Journal of Cellular Physiology,2017,232(10).

[40] Wang Si,Wang Xiuxia,Wu Yujian,Han Chunsheng. IGF-1R signaling is essential for the proliferation of cultured mouse spermatogonial stem cells by promoting the G2/M progression of the cell cycle[J]. Stem cells and development,2015,24(4).

[41] Khalil Ashraf,Jameson Mark J. Downregulation of IGF1R Expression Inhibits Growth and Enhances Cisplatin Sensitivity of Head and Neck Squamous Cell Carcinoma Cells In Vitro.[J]. Hormones \&amp; cancer,2019,10(1).

[42] Zhao Bing, Zheng Zebao. Insulin Growth Factor 1 Protects Neural Stem Cells Against Apoptosis Induced by Hypoxia Through Akt/Mitogen-Activated Protein Kinase/Extracellular Signal-Regulated Kinase (Akt/MAPK/ERK) Pathway in Hypoxia-Ishchemic Encephalopathy[J]. Medical science monitor: international medical journal of experimental and clinical research,2017,23. 
[43] Chunli Wang, Songcai Liu, Qingyan Wu, Yunyun Cheng, Tianqi Feng, Jie Song, Rui Yang, Hongwei Geng, Guanhong Lu, Siyao Wang, Linlin Hao. Porcine IGF-1R synonymous mutations in the intracellular domain affect cell proliferation and alter kinase activity[J]. International Journal of Biological Macromolecules,2020,152.

[44] Yu Pengfei, Ye Liang, Wang Hongbo, Du Guangying, Zhang Jianzhao, Zhang Jinghai, Tian Jingwei. NSK-01105 inhibits proliferation and induces apoptosis of prostate cancer cells by blocking the Raf/MEK/ERK and PI3K/Akt/mTOR signal pathways[J]. Tumour biology: the journal of the International Society for Oncodevelopmental Biology and Medicine,2015,36(3).

\section{Figures}

A

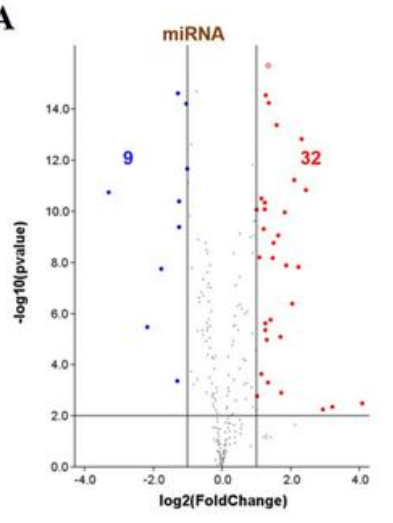

B

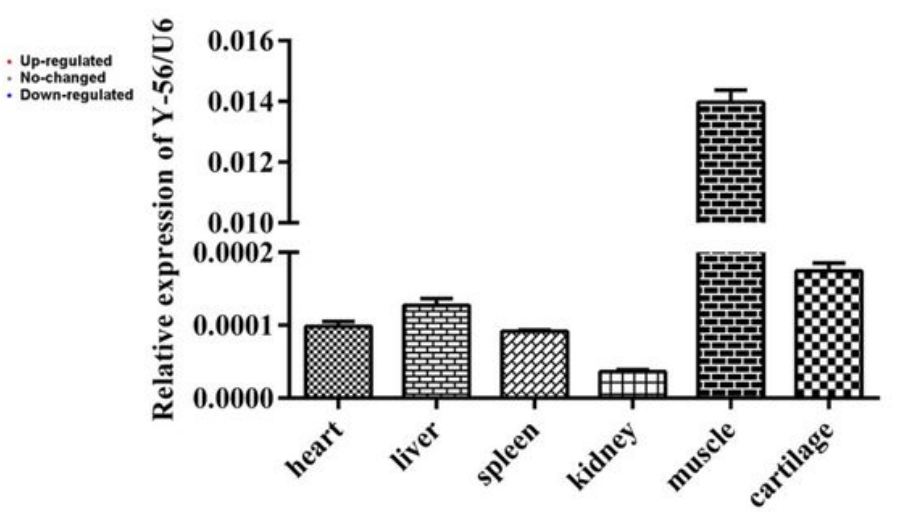

C

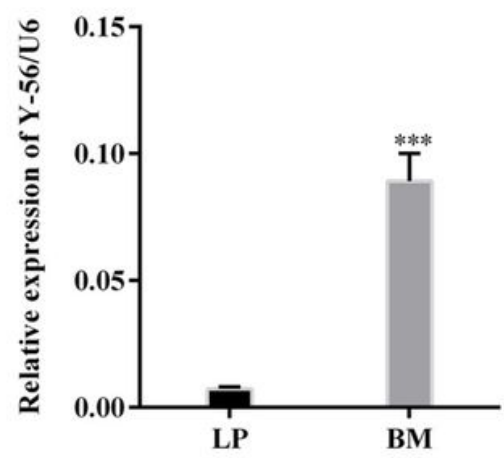

\section{Figure 1}

The expression level of Y-56 on porcine different tissues of the LP and the BM. 
A

B
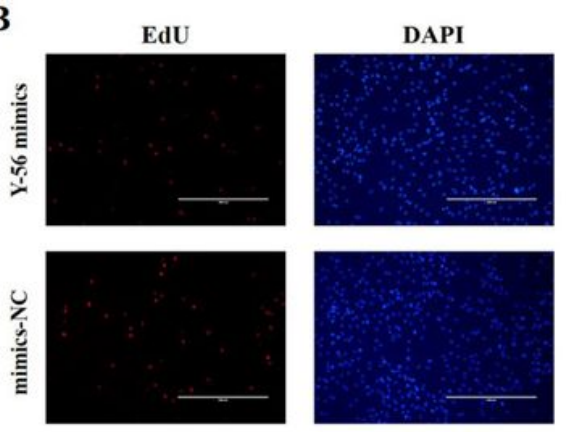

E Y-56 mimics

D
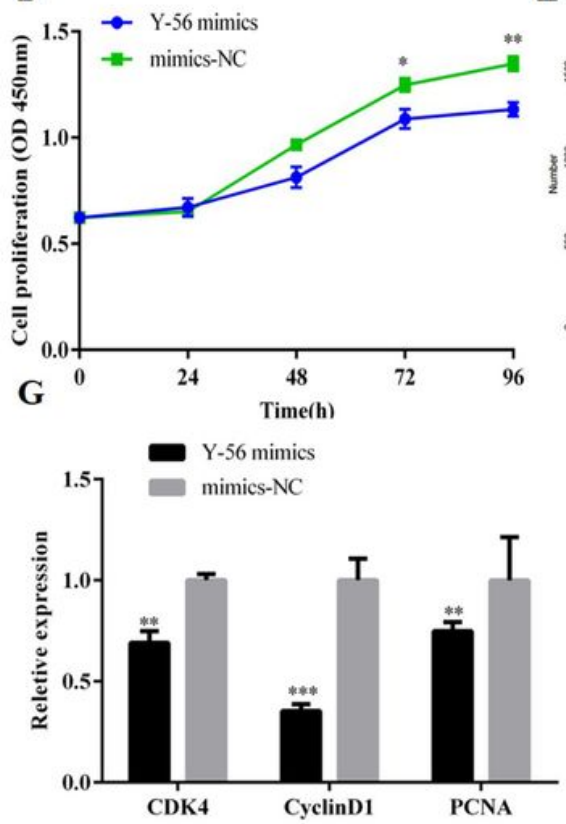
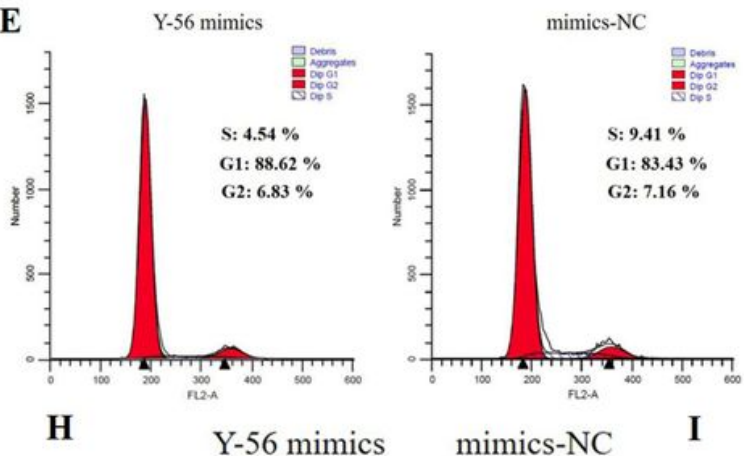

${ }^{1.5} 7$

CDK4

Cyclin D1

PCNA

$\beta$-actin

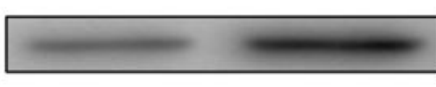

(20)

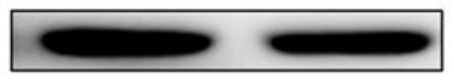

C
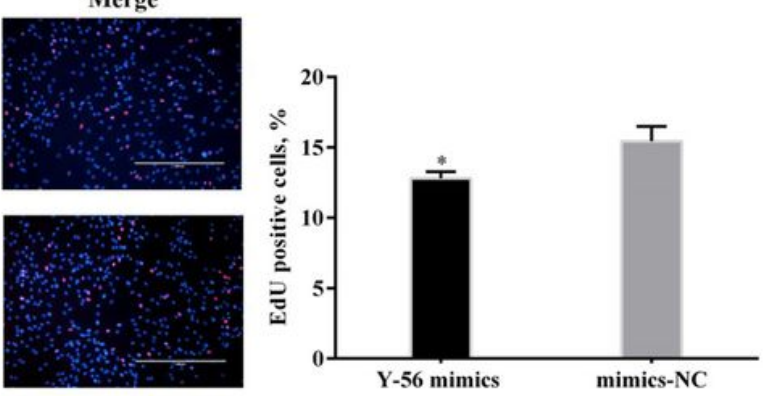

F

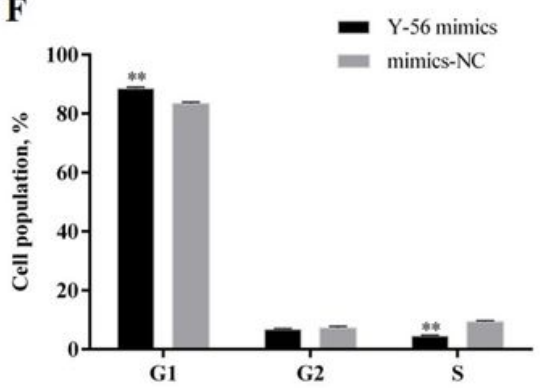

I-1. $\mathrm{Y}-56$ mimics

$=$ mimics-NC

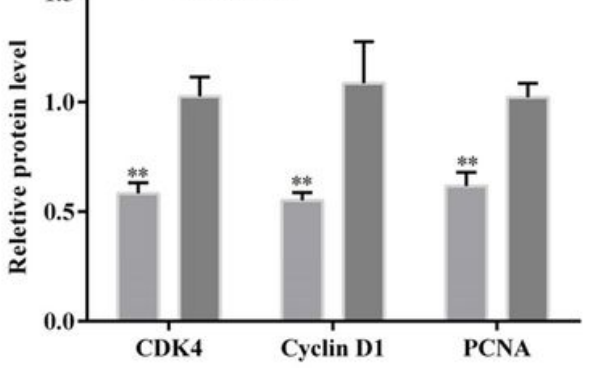

Figure 2

Overexpression of Y-56 suppressed the proliferation of the PSCs 
A

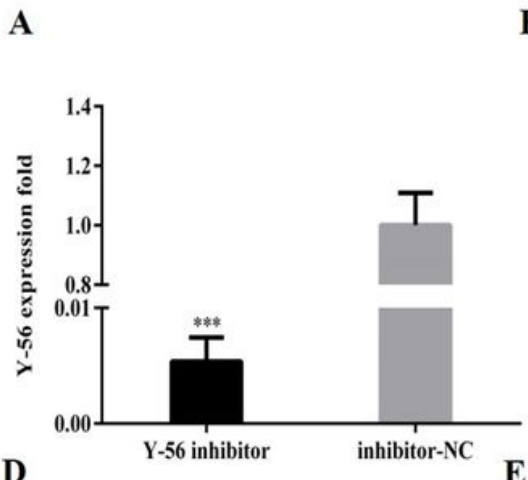

B

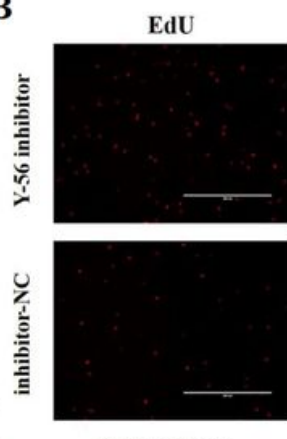

E Y-56 inhibitor

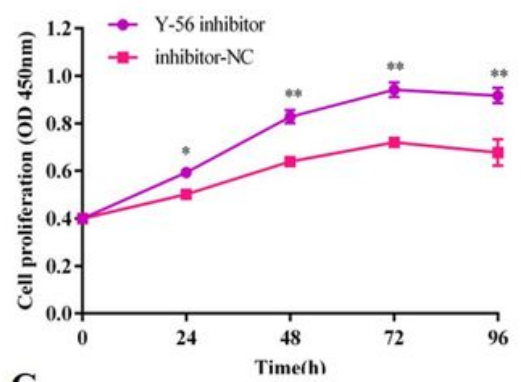

G

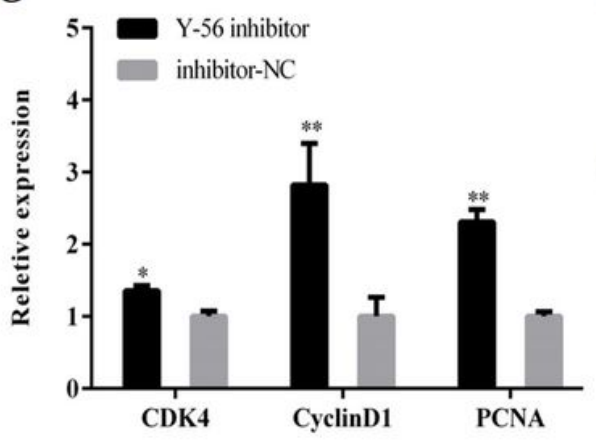

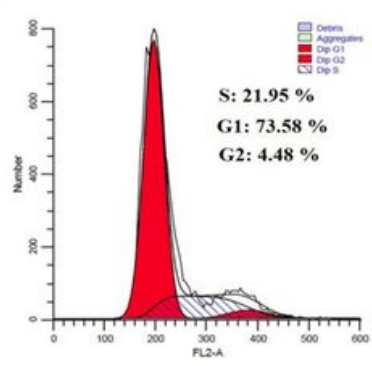

H
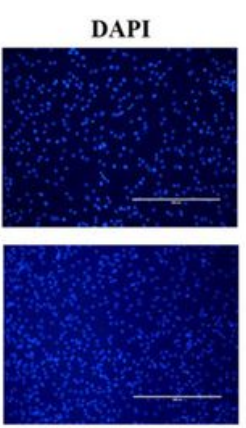

inhibitor-NC

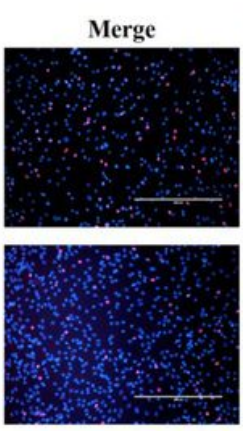

F
C

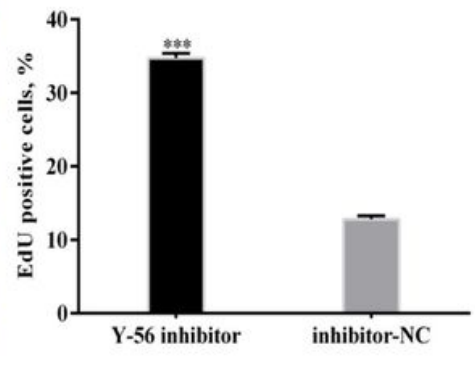

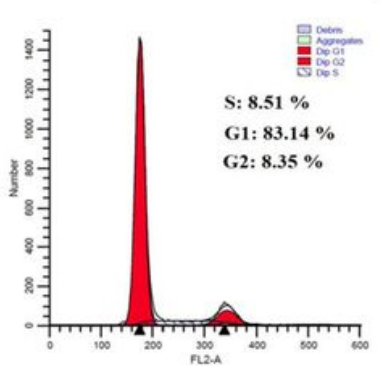

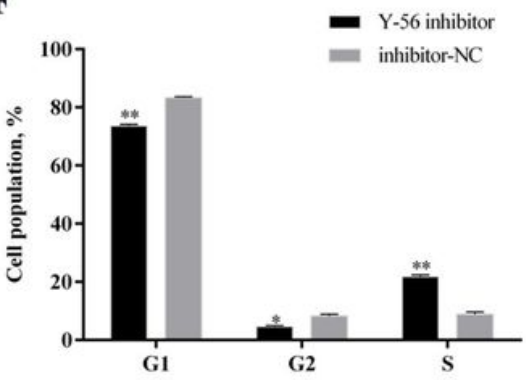

H $\quad$-56 inhibitor inhibitor-NC I

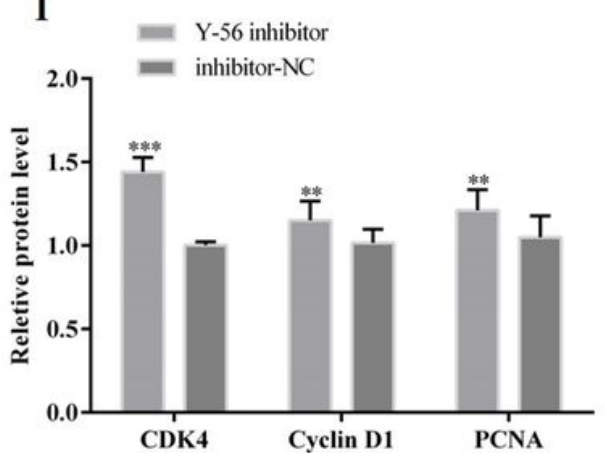

Figure 3

Inhibition of Y-56 promotes the cell proliferation of the PSCs 
A

IGF-1R 3'UTR WT 5'- CTTGAGAATCAAGCCTCCTCA -3'

Y-56 3'- AGGAGGAGGAGAAGGAGGAGA -5'

IGF-1R 3'UTR MT 5'- CTTGAGAATCAAGAAGATCAA -3'

C
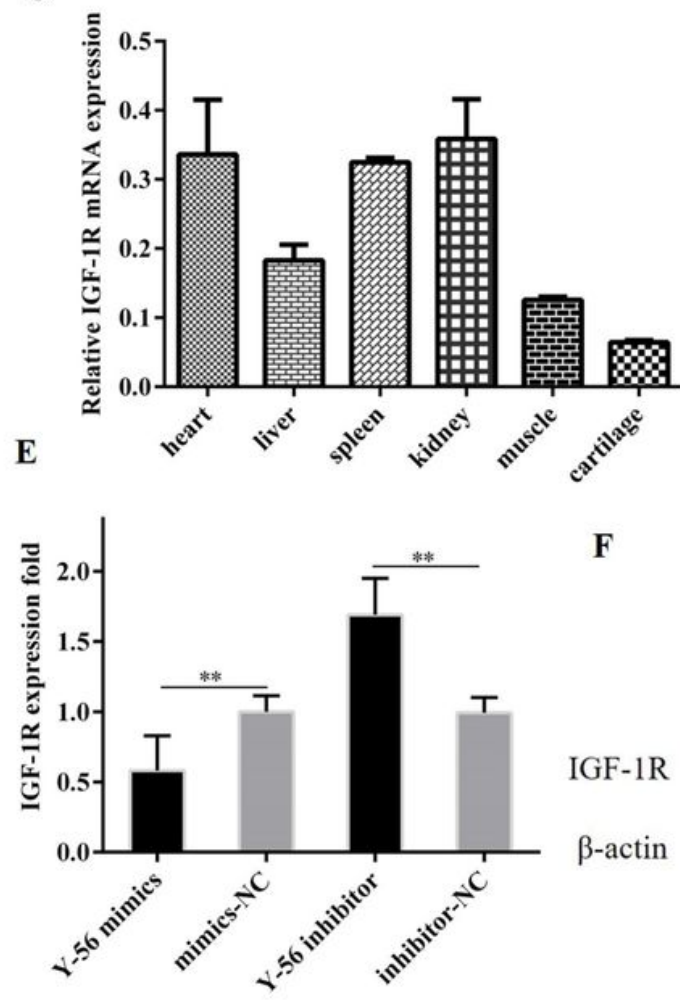

111111

D

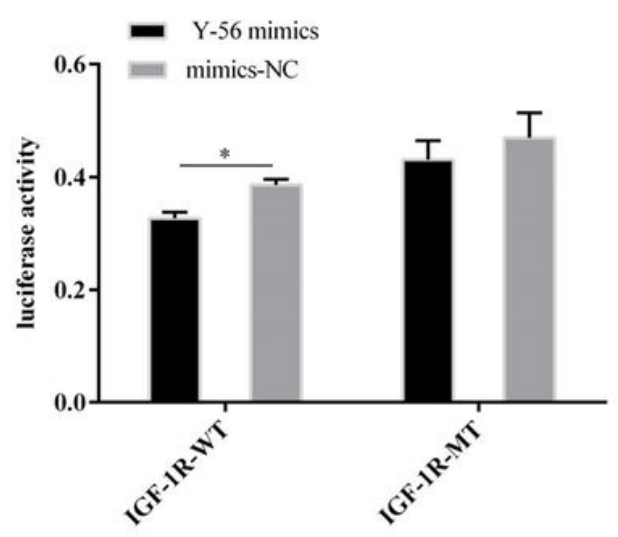

B

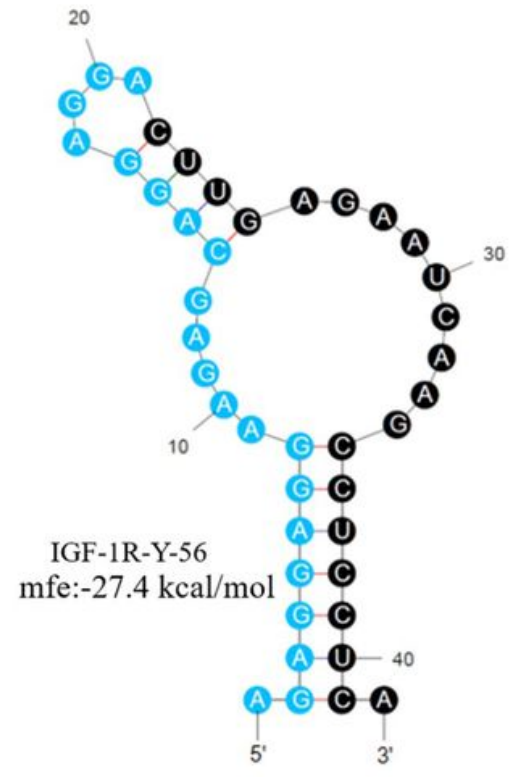

G
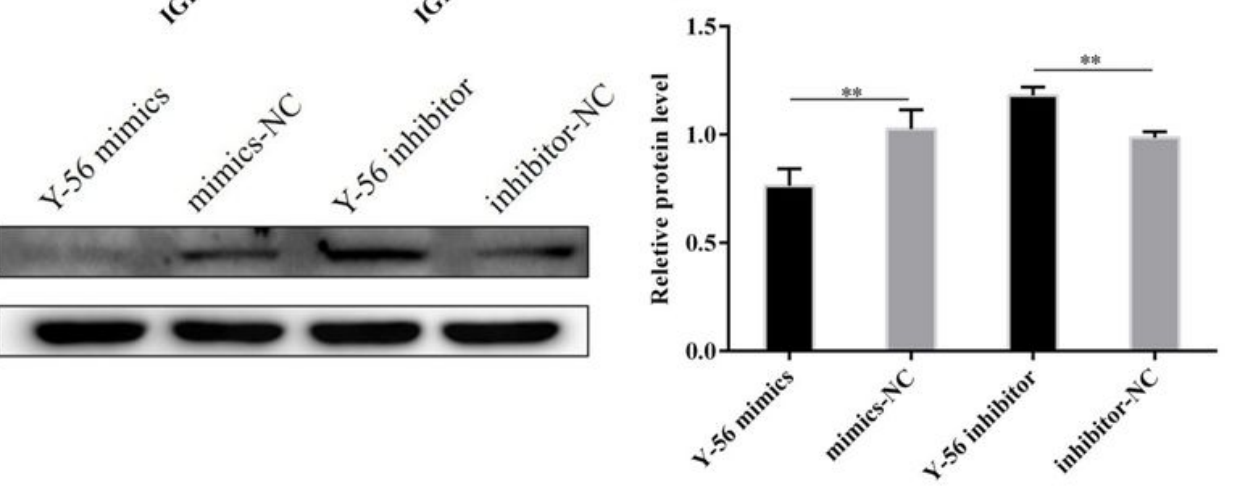

Figure 4

IGF-1R is a direct target gene of $\mathrm{Y}-56$ 

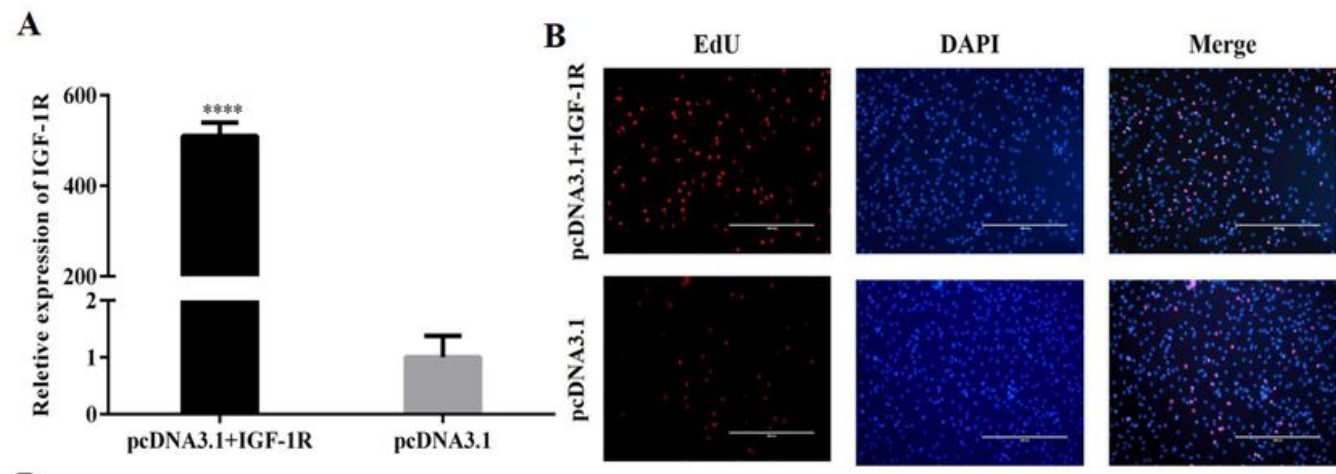

C

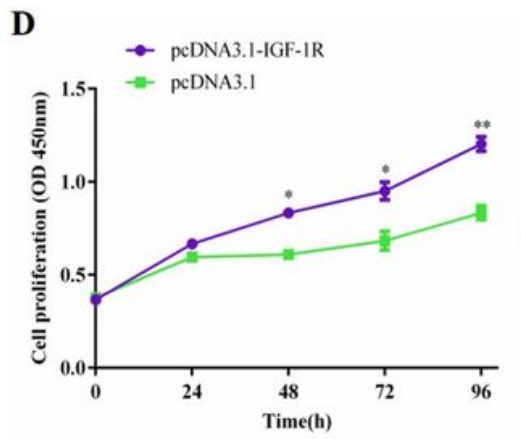

E peDNA3.1+IGF-1R
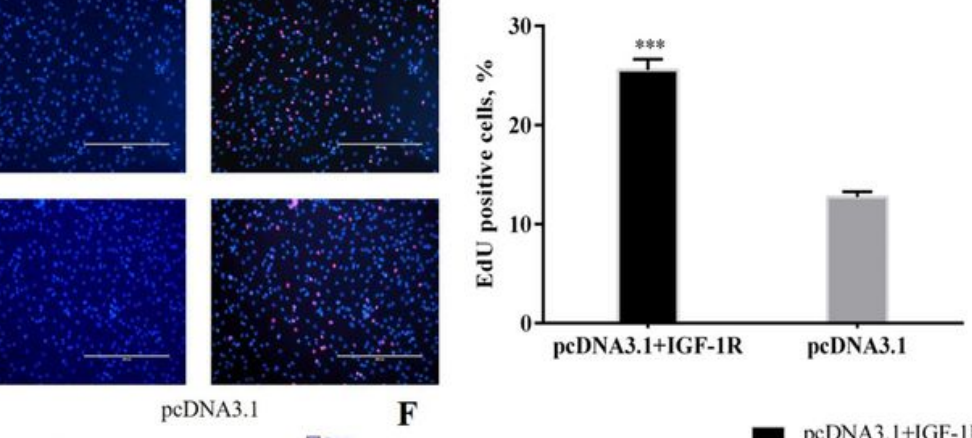

G
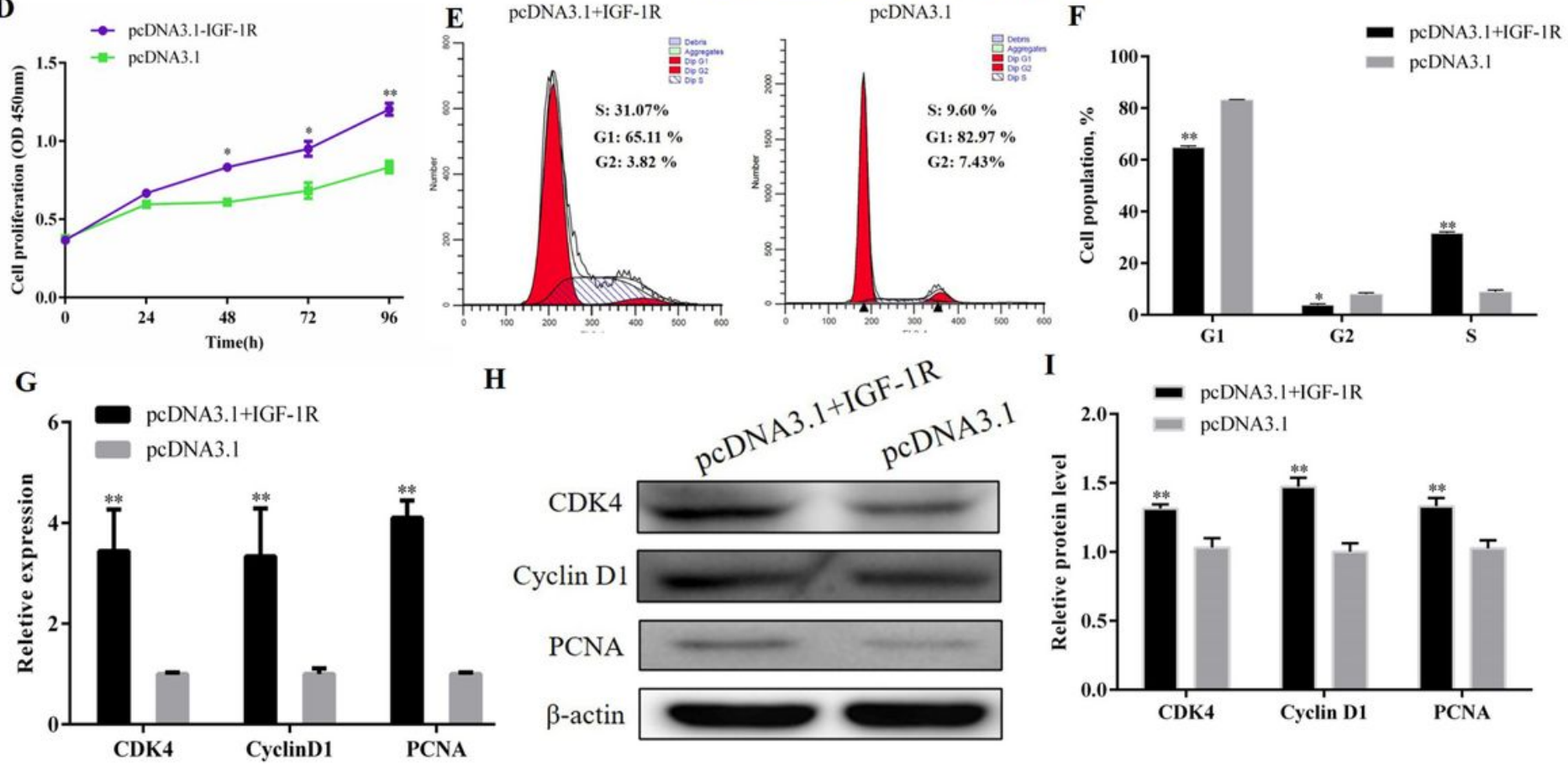

$\mathbf{H}$

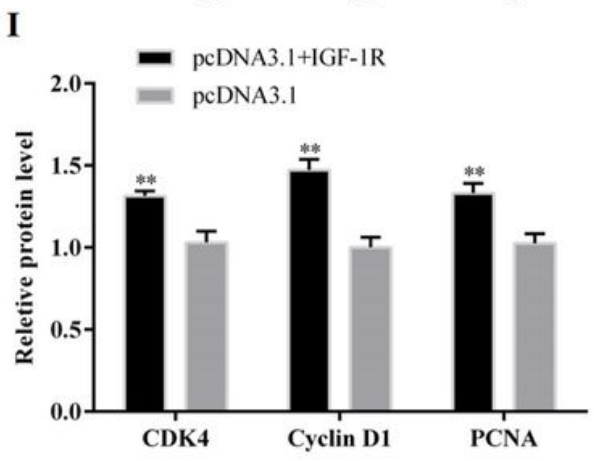

Figure 5

IGF-1R facilitates the cell proliferation of the PSCs 


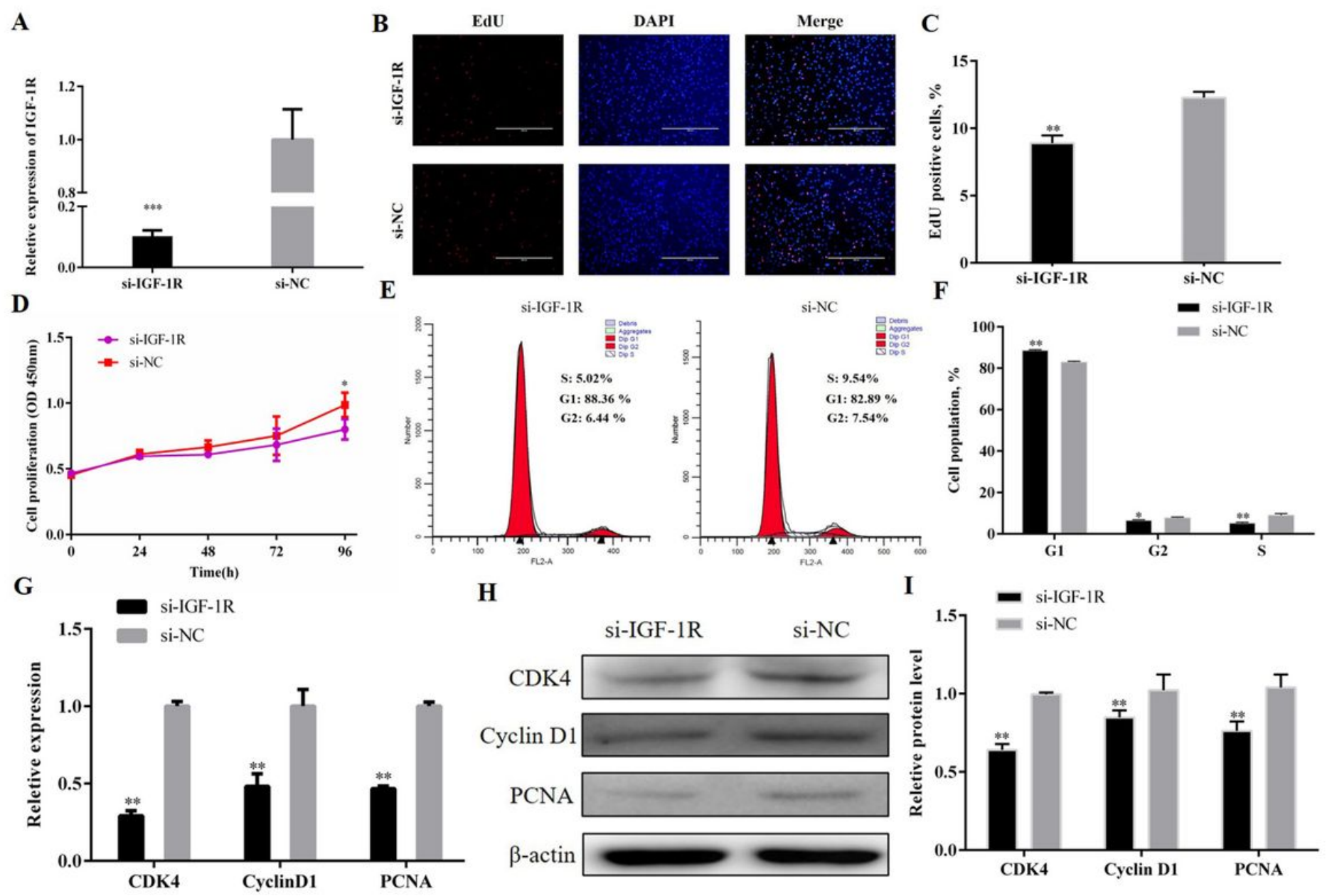

Figure 6

Knock-down IGF-1R reduces cell proliferation of the PSCs 

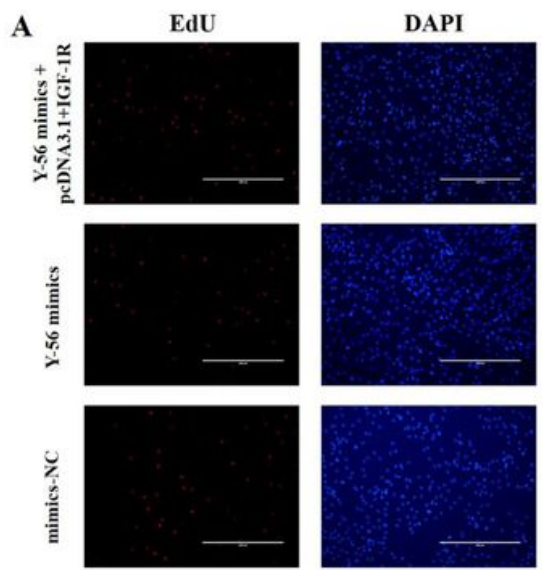

D
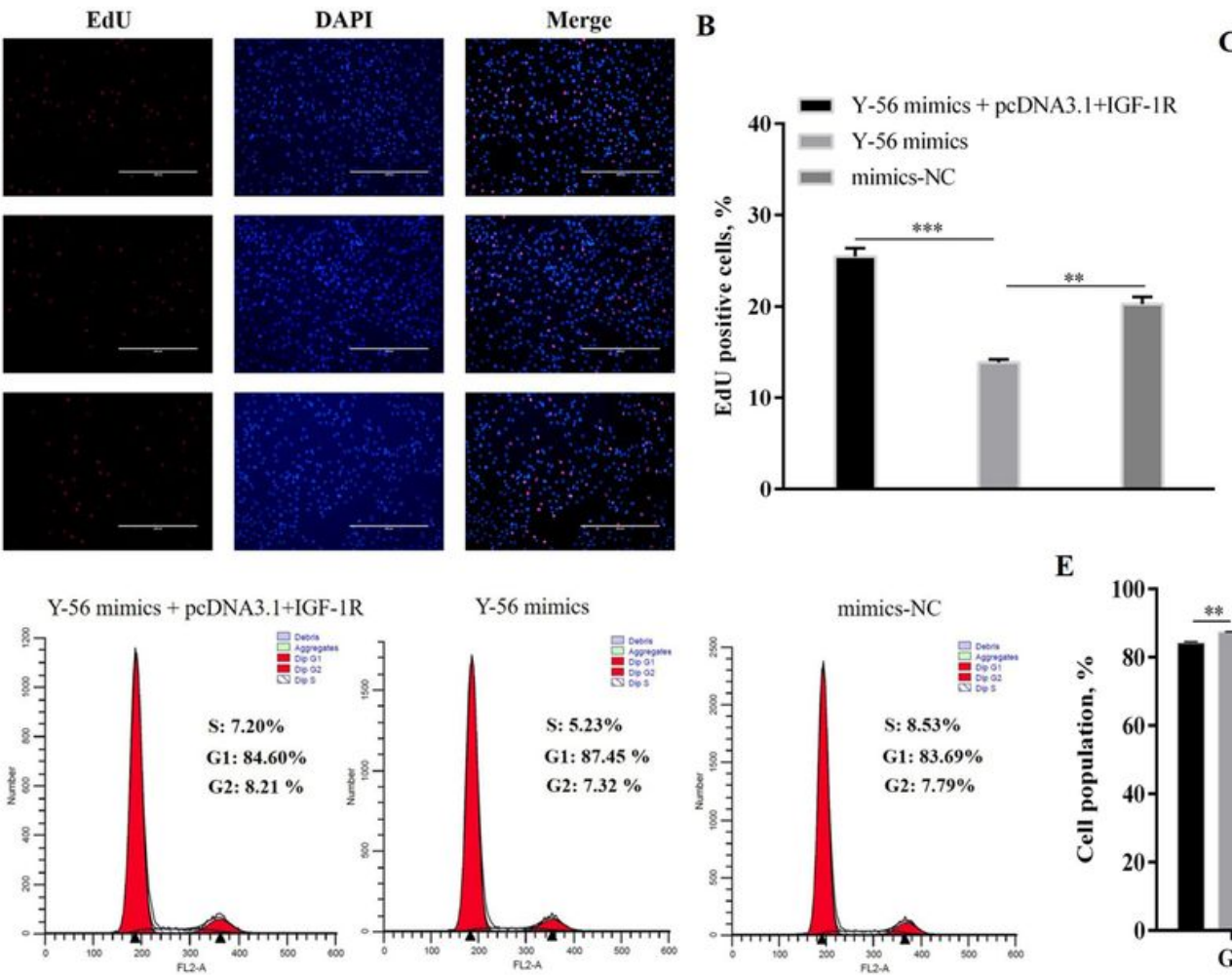

F

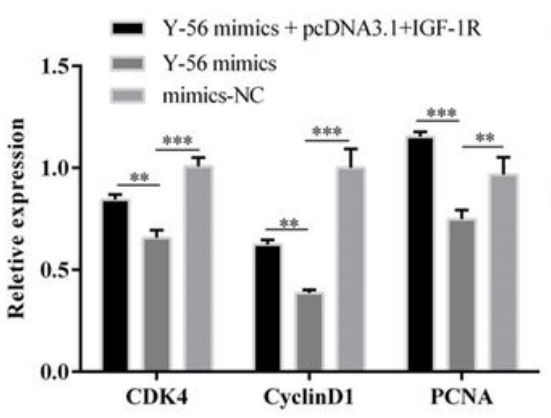

G

G Y-56 mimics +

CDK4

Cyclin D

PCNA pcDNA3.1+IGF-1R

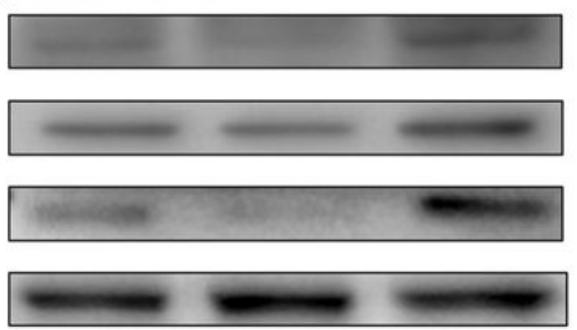

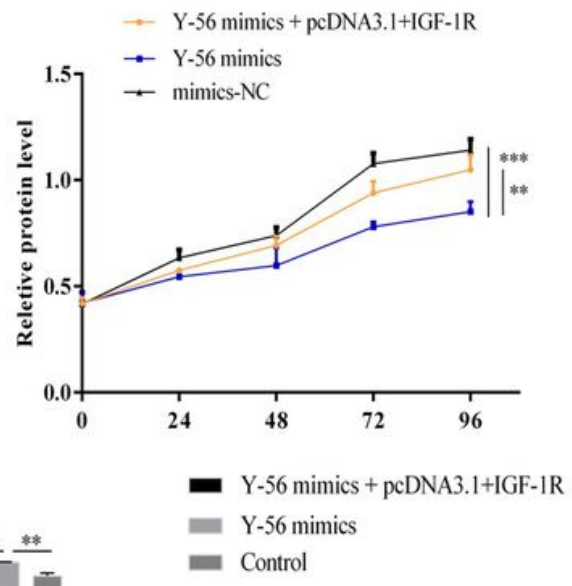

C

- Y-56 mimics + pcDNA3.1+IGF-IR

G2

$\mathrm{H}=\mathrm{Y}-56$ mimics

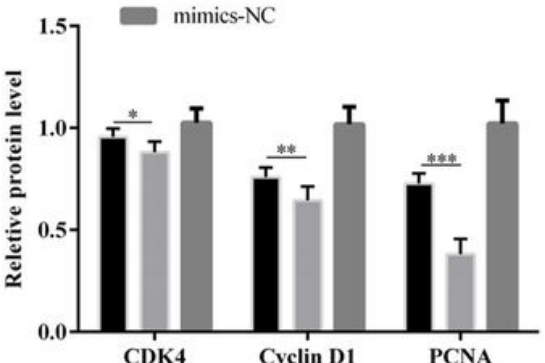

Figure 7

IGF-1R reverses the inhibitory effect of Y-56 on the PSCs 


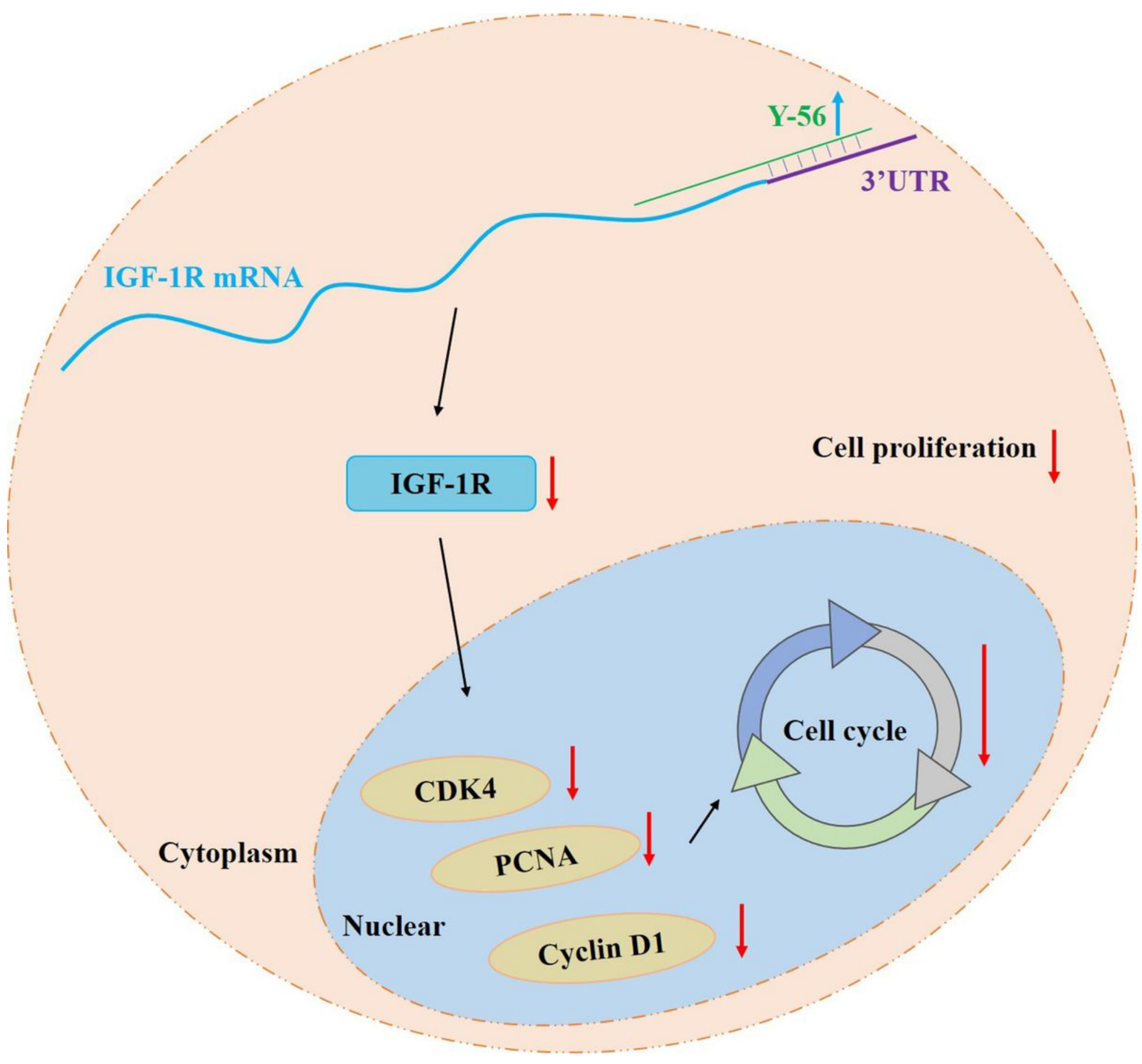

Figure 8

Schematic model of Y-56 regulation on the PSCs proliferation

\section{Supplementary Files}

This is a list of supplementary files associated with this preprint. Click to download.

- Fig.S1.jpg 